\title{
Ausschreibungen 2022 der Sektion Kopf-Hals und des Arbeitskreises Mammasonografie
}

Förderpreis der Sektion

\section{Kopf-Hals}

Die Sektion Kopf-Hals der Deutschen Gesellschaft für Ultraschall in der Medizin (DEGUM) schreibt auch für 2022 den Förderpreis der Sektion aus. Der Preis ist mit 500 Euro dotiert und richtet sich an Wissen- schaftler*innen, die sich mit außergewöhnlichen Publikationen oder einer Habilitationsschrift der Kopf-Hals-Sonografie verdient gemacht haben. Bewerbungsfrist ist der 30.04.2022. Die Preisverleihung erfolgt während eines Treffens der Sektion Kopf-Hals im zweiten Halbjahr 2022, entweder in Präsenz oder virtuell.
Förderpreis des Arbeitskreises Mammasonografie

Der Arbeitskreis Mammasonografie der Deutschen Gesellschaft für Ultraschall in der Medizin (DEGUM) schreibt auch für 2022 den Förderpreis des Arbeitskreises aus. Der Preis ist mit 500 Euro dotiert und 
richtet sich an Wissenschaftler*innen der Mammasonografie, die sich mit außergewöhnlichen Publikationen oder einer Habilitationsschrift der Mammasonografie verdient gemacht haben. Bewerbungsfrist ist der 15.03.2022. Die Preisverleihung erfolgt im Jahr 2022 während eines Treffens des Arbeitskreises.

\section{Für beide Förderpreise gilt}

Autor*innen, die bereits einen DEGUM-Preis verliehen bekommen haben, können sich nicht ein zweites Mal bewerben. Es ist jedoch möglich, dass eine abgelehnte Arbeit im Folgejahr erneut eingereicht wird.

Die Anträge sowohl für den Förderpreis der Sektion Kopf-Hals als auch für den Förderpreis des Arbeitskreises Mammasonografie sind formlos auf elektronischem Weg unter Anlage der Publikation (veröffentlicht oder akzeptiert zur Veröffentlichung mit Nachweis des Journals) bzw. der Habilitationsschrift an die DEGUMGeschäftsstelle (DEGUM e. V., Charlot- tenstr. 79/80, 10117 Berlin, geschaeftsstelle @degum.de) zu richten.

Die Anwesenheit des Preisträgers bzw. der Preisträgerin bei den Jahrestreffen der Sektion bzw. des Arbeitskreises ist obligat. Im Rahmen der Preisverleihung wird der Preisträger bzw. die Preisträgerin aufgefordert, die prämierte Arbeit in einem Kurzreferat vorzustellen. 